Journal of Astronomical Instrumentation, Vol. 7, No. 4 (2018) 1840004 (16 pages)

(C) The Author(s)

DOI: $10.1142 / \mathrm{S} 2251171718400044$

\title{
Spectral and Spatial Characterization and Calibration of FIFI-LS - The Field Imaging Spectrometer on SOFIA
}

\author{
Sebastian Colditz ${ }^{1,12}$, Simon Beckmann ${ }^{1}$, Aaron Bryant ${ }^{2}$, Christian Fischer ${ }^{1}$, \\ Fabio Fumi ${ }^{2}$, Norbert Geis ${ }^{3}$, Mourad Hamidouche ${ }^{4}$, Thomas Henning ${ }^{5}$, Rainer Hönle ${ }^{2}$, \\ Christof Iserlohe ${ }^{1}$, Randolf Klein ${ }^{6}$, Alfred Krabbe ${ }^{1,2}$, Leslie Looney ${ }^{7}$, Albrecht Poglitsch ${ }^{3}$, \\ Walfried Raab ${ }^{8}$, Felix Rebell ${ }^{2}$, Dirk Rosenthal ${ }^{9}$, Maureen Savage ${ }^{6,10}$, \\ Mario Schweitzer ${ }^{11}$ and William Vacca ${ }^{6}$ \\ ${ }^{1}$ Deutsches SOFIA Institut, University of Stuttgart \\ 70569 Stuttgart, Germany \\ ${ }^{2}$ Institute of Space Systems, University of Stuttgart \\ 70569 Stuttgart, Germany \\ ${ }^{3}$ Max Planck Institute for Extraterrestrial Physics \\ 85748 Garching, Germany \\ ${ }^{4}$ Remote Sensing Technology Institute \\ DLR (German Aerospace Center), 82234 Wessling, Germany \\ ${ }^{5}$ Max Planck Institute for Astronomy \\ 69117 Heidelberg, Germany \\ ${ }^{6}$ SOFIA-USRA, NASA Ames Research Center \\ Moffett Field, CA94035, USA \\ ${ }^{7}$ Department of Astronomy, University of Illinois \\ Urbana, IL, 61801, USA \\ ${ }^{8}$ ESTEC, European Space Agency, 69117 \\ Nordwijk, 2201 AZ, The Netherlands \\ ${ }^{9}$ OSRAM GmbH, Industriestraße 20B \\ 85072 Eichstätt, Germany \\ ${ }^{10}$ UC Observatories, 1156 High Street \\ Santa Cruz, CA, 95064, USA \\ ${ }^{11}$ OHB System AG, 822234 Wessling, Germany \\ ${ }^{12}$ colditz@dsi.uni-stuttgart.de
}

The field-imaging far-infrared line spectrometer (FIFI-LS) is a science instrument for the Stratospheric Observatory for Infrared Astronomy (SOFIA). FIFI-LS allows simultaneous observations in two spectral channels. The "blue" channel is sensitive from $51 \mu \mathrm{m}$ to $125 \mu \mathrm{m}$ and the "red" channel from $115 \mu \mathrm{m}$ to $203 \mu \mathrm{m}$. The instantaneous spectral coverage is $1000-3000 \mathrm{~km} / \mathrm{s}$ in the blue and $800-2500 \mathrm{~km} / \mathrm{s}$ in the red channel with a spectral resolution between $150 \mathrm{~km} / \mathrm{s}$ and $600 \mathrm{~km} / \mathrm{s}$. Each spectral channel observes a field of five by five spatial pixels on the sky. The pixel size in the blue channel is 6.14 by 6.25 square arc seconds and it is 12.2 by 12.5 square arc seconds in the red channel. FIFI-LS has been operating on SOFIA since 2014. It is available to the astronomical community as a facility science instrument. We present the results of the spectral and spatial characterization of the instrument based on laboratory measurements. This includes the measured spectral resolution and examples of the line spread function in the spectral domain. In the spatial domain, a model of the instrument's point spread function (PSF) and the description of a second pass ghost are presented. We also provide an overview of the procedures used to measure the instrument's

This is an Open Access article published by World Scientific Publishing Company. It is distributed under the terms of the Creative Commons Attribution 4.0 (CC-BY) License. Further distribution of this work is permitted, provided the original work is properly cited. 


\section{S. Colditz et al.}

field of view geometry and spectral calibration. The spectral calibration yields an accuracy of $15-60 \mathrm{~km} / \mathrm{s}$ depending on wavelength.

Keywords: Integral field spectroscopy, spectrometer, far-infrared, FIFI LS, FIFI-LS, SOFIA.

\section{Introduction}

The Stratospheric Observatory for Infrared Astronomy (SOFIA) is a highly modified Boeing 747 aircraft carrying a $2.5 \mathrm{~m}$ telescope (Erickson \& Davidson, 1993; Young et al., 2012). SOFIA enables observations of far-infrared radiation from celestial sources by flying at altitudes of up to $13.7 \mathrm{~km}$. This flight level is above $99.8 \%$ of the atmospheric water vapor, which absorbs most of the far-infrared radiation at ground-based observatories (Krabbe et al., 2013). Typically, the observatory can accommodate one instrument at its primary focus on a specific flight. One of these instruments is the field-imaging far-infrared line spectrometer (FIFI-LS) which has been specifically designed for SOFIA. FIFI-LS is an imaging spectrometer with two spectral channels allowing simultaneous observations in the two spectral bands. The short wavelength band of the instrument's "blue" channel is sensitive from 51 to $125 \mu \mathrm{m}$, while the long wavelength band of the "red" channel may be used for observations from 115 to $203 \mu \mathrm{m}$. The blue channel spectral band is further divided into two sub-bands. The first band uses the first diffraction order of the blue channel's grating, from $71 \mu \mathrm{m}$ to $125 \mu \mathrm{m}$. The second band uses the second diffraction order, from 51 to $71 \mu \mathrm{m}$. The active band is selected by a filter mechanism that allows switching between the two bands in flight.

Each channel has a five by five spatial pixel (spaxel) field of view (FOV) on the sky. The FOVs of the two channels are approximately concentric the offset was measured as part of the instrument



Slit direction, increasing slit position
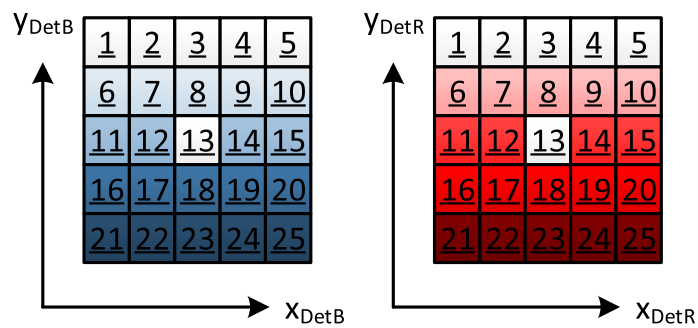

Fig. 1. Detector numbering conventions. Top: Schematic view of a 25 by 16 pixel detector. The spaxel/module numbers are underlined above the schematic. Each module has a slit position number assigned to it, shown in bold below the schematic. These numbers are used for the wavelength calibration. The individual pixel numbers are in italics in the detector pixel boxes. When referring to a pixel within a specific spaxel, the pixel numbering for spaxel 1 is used. For example, pixel 17 may also be denominated pixel 1 of spaxel 2. Bottom: Spatial view of the detector spaxels in the FOV, including the respective detector coordinate systems. The spaxel/module numbers are underlined in the spaxel boxes. The origin of each coordinate system is at the center of the respective spaxel 13 . 
characterization. The spaxel size of the blue channel is 6.14 by 6.25 square arc seconds and the spaxel size of the red channel is 12.2 by 12.5 square arc seconds. Both FIFI-LS detectors are made of 25 modules of 16 physical pixels using one module for each spaxel. Each of the 16 pixels in one module samples a spectral width of about 50 to $190 \mathrm{~km} / \mathrm{s}$ (the exact values depend on the specific channel and the observed wavelength). Figure 1 shows the numbering scheme used for the individual spaxels and physical pixels of the FIFI-LS detectors [refer to Fischer et al., 2018 for a description of the field-imaging concept of FIFI-LS].

By design, FIFI-LS has simultaneous characteristics of a spectrometer and an imager at the same time. Therefore, this paper splits the description of the instrument characteristics into two parts. In Sec. 2, we focus on the spectral characteristics and calibration of the instrument while in Sec. 3 we discuss the instrument characteristics from the imager perspective, focusing on spatial effects. An overview of the FIFI-LS instrument operation and performance (e.g. sensitivity) is given by Fischer et al. (2018). The calibration results summarized in this paper are discussed in further detail in Colditz (2017).

\section{Spectral Characterization and Calibration}

The spectral characterization of the instrument in the laboratory was achieved using a telescope simulator specifically designed for the FIFI-LS Instrument (Schweitzer, 2008). This simulator contains a gas cell in front of a nitrogen cold $(77 \mathrm{~K})$ black background. The gas cell was filled with ambient air containing ambient water vapor or with pure water vapor. The total pressure in the gas cell was set to values of 1,5 or 10 mbar. The water vapor provided several measurable spectral emission lines. The measurements were made by scanning the spectra over the detector using small stepwise motions of the diffraction grating in each channel. The step size was chosen smaller than one half of the smallest spectral full width at half maximum (FWHM) to ensure Nyquist-sampling. This size corresponded to a move of the spectrum on the detector of roughly one third of a spectral pixel.

\subsection{Line spread function}

The expected shape of the instrument's line spread function (LSF) can be estimated with a Gaussian according to the results of the scalar diffraction analysis of the instrument presented in Raab (2002). Gaussian and Lorentzian models were fitted to the measured water emission lines to evaluate this assumption. The fit results (Fig. 2, left side) in the red channel show that the Gaussian model fits the data quite well and with lower residual errors than the Lorentzian model. The left of the figure shows the data from a single physical pixel for the different grating positions. The grating positions have already been converted to wavelength based on the wavelength calibration presented in Sec. 2.3. The line profile is not affected by the conversion because in the close vicinity of a line, the conversion can be approximated by a constant conversion factor as shown in Colditz (2017).

The LSF diverges slightly from the Gaussian model on the short wavelength side of the line. The right side of Fig. 2 shows that the LSF does not differ significantly for the 16 spectral pixels of one detector module (or spaxel). The standard deviation of the line width parameter $\sigma_{\lambda}$ over all pixels of the red detector and within each module was less than $10 \%$ of its mean value. Data from lines with strong pressure broadening were evaluated to ascertain that the measurements produced correct line shapes. These measurements of resolved lines had clear Lorentzian profiles as expected for pressurebroadened lines.


Fig. 2. Example of the red channel's LSF for the $132.4084 \mu \mathrm{m}$ water emission line with air at 10 mbar in the gas cell. Left: model fits to the data of a single spectral pixel of the central spaxel (pixel 8 in spaxel 13). Right: measured data for all 16 pixels of the central spaxel 13, with the baseline subtracted and the peak heights normalized to 1 (Colditz, 2017). The grating position has been converted to wavelength on the $x$-axis, based on the wavelength calibration introduced in Sec. 2.3. 




Fig. 3. Example of the blue channel's LSF in first diffraction order for the $93.3829 \mu \mathrm{m}$ water emission line with pure water vapor at 5 mbar in the gas cell. The LSF is Gaussian only for pixels 16 and 15. Pixels with lower pixel numbers show a strong deviation from a Gaussian. The pixels closer to the center (e.g. pixel 10) almost show a second line peak. The intensity curves shown are for pixels of the central spaxel in the blue channel and are normalized to their peak height. The curves are shifted such that the short wavelength sides of the curves are on top of each other (Colditz, 2017).

In the blue channel, the LSF is Gaussian only for the pixels 15 and 16 in each module, according to the numbering scheme in Fig. 1. For decreasing pixel numbers, the LSF deviates from a Gaussian, gradually deforming towards the center of a module. The deformation decreases again with decreasing pixel number away from the detector center. However, the LSF is still not Gaussian in pixel 1. The deformation occurs on the long wavelength side of a line. It looks like a second peak for the central pixels of a module, which are affected most.

Figure 3 shows the data from a measurement of the $93.3829 \mu \mathrm{m}$ water emission line in the first order of the blue channel. This measurement was obtained with pure water vapor at a pressure of about 5 mbar within the gas cell. The line was unresolved, having a simulated FWHM of ca. $0.03 \mu \mathrm{m}$ according to "HITRAN on the web" (HITRAN, 2016), which was a third of the theoretically estimated FWHM of the instrument's LSF at this wavelength (i.e. $0.09 \mu \mathrm{m}$ ).

The strong dependence of the LSF on the pixel number is clearly visible on the long wavelength side in Fig. 3. For observations in the first grating order, the deformation caused a standard deviation of the fitted Gaussian $\sigma_{\lambda}$-widths of about $20 \%$ of the mean value over all 400 pixels of the blue detector. Qualitatively, the deformation was not as severe in the blue channel's second order, but still caused a standard deviation of the $\sigma_{\lambda}$-values of roughly $15 \%$ around the mean value over all 400 pixels. The deformation of the LSF has to be investigated further in order to derive a pixel and wavelength dependent model and ultimately, to find the cause of the deformation.

\subsection{Spectral resolution from laboratory measurements}

The spectral resolution of the instrument was determined using the fitted $\sigma_{\lambda}$-values from measurements made with air at 10 mbar in the gas cell. Only unresolved spectral lines were considered for these measurements. The FWHM of a line was approximated using the median of $\sigma_{\lambda}$ over all 400 pixels of a detector (excluding bad pixels) and transforming it assuming a Gaussian profile. Figure 4 shows a comparison between the theoretical estimation of the resolving power and the resolving power approximated with the gas cell measurements. The theoretical estimates are based on the root mean square sum of the projected slit width at the detector and the theoretical grating resolution. The pixel sampling widths, the diffraction effects originating in the imager slicer, and the effects of optical imperfections were not considered in this estimation. All these effects tend to widen the spectral profiles and reduce the resolving power.

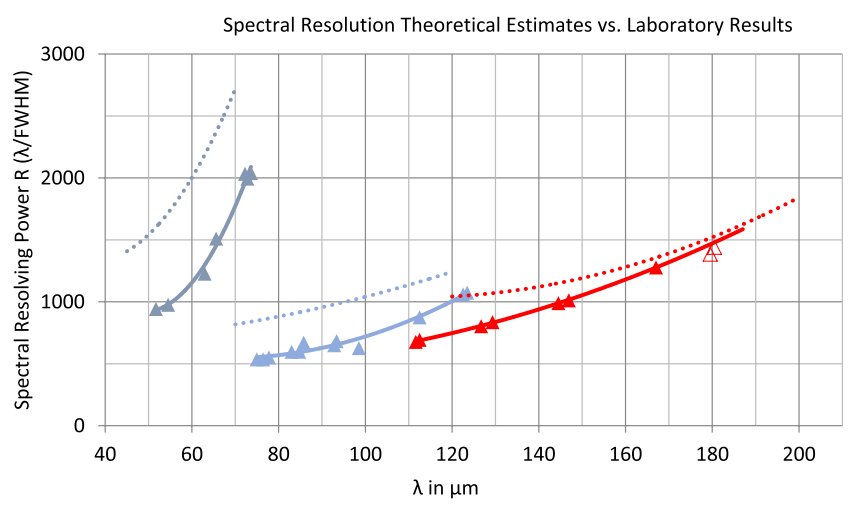

Fig. 4. The dotted lines show the estimated resolving power for extended sources. The estimated curves are given for the blue channel second order in dark blue, blue channel first order in light blue and the red channel in red. The triangles in the respective colors show the fitted resolving power for line measurements using the gas cell filled with air at 10 mbar pressure. The solid lines show polynomial fits (second-order polynomials) for the red channel, and the blue channel's first and second order. The data points for the hollow red triangles in the red channel were not used for the fit, because these lines might be wide enough to be resolved, but they show a lower limit for the resolving power (Colditz, 2017). 
The dependencies of the blue channel's first and second order resolving powers on the wavelength were approximated by the second order polynomial fits in Eq. (1).

$$
\begin{aligned}
& R_{\text {Blue }, 1^{\text {st } o r d e r}}(\lambda)=0.169 \lambda^{2}-22.8 \lambda+1320 \\
& R_{\text {Blue }, 2^{\text {nd }} \text { order }}(\lambda)=1.92 \lambda^{2}-187 \lambda+5500 .
\end{aligned}
$$

The values of these fits are $\sim 15-35 \%$ lower than the estimated values for the first order and $\sim 30-45 \%$ lower for the second order. Part of this reduction in resolving power is related to the deformation of the blue channel's line profiles presented in the previous sub-section.

The dependence of the red channel's resolving power on the wavelength was approximated by the second-order polynomial fit Eq. (2).

$$
R_{\text {Red }}(\lambda)=0.0624 \lambda^{2}-6.66 \lambda+650 .
$$

The value of this fit is $\sim 35 \%$ below the estimated value at a wavelength of $100 \mu \mathrm{m}$. The differences between the fitted resolving power and the estimated resolving power decrease for increasing wavelengths. At roughly $185 \mu \mathrm{m}$, the measured FWHM values are in the range expected from the estimates. The FWHM values fitted from the measured data and the estimated $R$-values were converted into pixel scale to analyze why the red channel's resolving power was lower than expected. The results of these conversions are plotted in Fig. 5. This figure shows that the FWHM of lines should increase in pixel scale with increasing wavelength according to the estimated data, but the measured data shows that the FWHM is almost constant at 2.7 pixels for the whole wavelength range below $170 \mu \mathrm{m}$. This increased FWHM has to be investigated further. A possible cause is that the

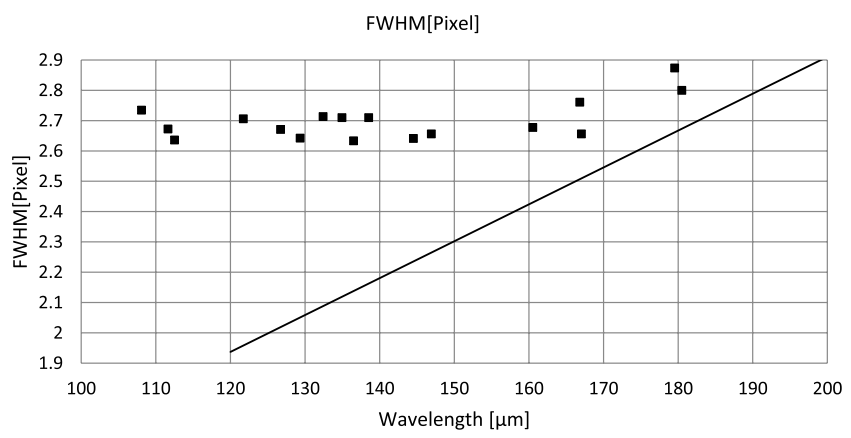

Fig. 5. Red channel FWHM in pixel scale. The solid line shows a linear fit to the estimated FWHM in pixel scale. The squares show the FWHMs resulting from the fits to the measured data (Colditz, 2017). beam from the grating to the detector is defocused in the wavelength direction and that this defocus dominates the width of the LSF leading to the almost constant width in Pixel space shown in Fig. 5.

\subsection{Spectral calibration}

The spectral calibration relates the angular position of the diffraction grating to the wavelength observed at a physical pixel on the detector. The calibration is therefore of major importance for the control of the instrument (i.e. to make observations at the correct wavelength) and for the interpretation of the data (i.e. to assign the measured flux to its source wavelength). The wavelength calibration was derived individually for each of the three spectral bands of the instrument: one band in the red channel from 115 to $203 \mu \mathrm{m}$, and two bands in the blue channel for the two diffraction orders from 51 to $71 \mu \mathrm{m}$ and from 71 to $125 \mu \mathrm{m}$.

A calibration model has been derived starting from the standard grating Eq. (3) (Voigt, 2012).

$$
\lambda=\frac{g}{m}(\sin \Phi+\sin \Theta) .
$$

In this equation, $g$ is the groove spacing of the grating. The symbol $m$ is the observed diffraction order. The angle $\Phi$ is the angle of incidence of light on the grating, and the angle $\Theta$ is the angle of the observed outgoing beam. Equation (3) was adapted to account for the specific optical setup of FIFI-LS, yielding Eq. (4).

$$
\lambda=\frac{g}{m}(\sin (\varphi-\gamma)+\sin (\varphi+\gamma+\delta)) .
$$

Here, $\varphi$ is the ideal Littrow angle (Voigt, 2012). In FIFI-LS, the inbound and outbound beams divert slightly from the ideal Littrow angle by the angle $\gamma$ in either direction, forming a "QuasiLittrow" setup. The pixel angle $\delta$ in the term for the outbound beam accounts for the extended detector and is dependent on the individual pixel position (1-16) within one detector module. The pixel angle $\delta$ is $0^{\circ}$ for a beam heading toward the center of the detector.

The grating is rotated to select the observed wavelength range. This rotation changes the value of the Littrow angle $\varphi$. The angular position of the grating is measured with a rotary Inductosyn ${ }^{\circledR}$ transducer manufactured by FARRAND (3.94 inch, 512 poles). The resolution per readout element is ca. 0.077 arcseconds (Raab, 2002). The digital value 
from the transducer is converted to the angle $\Delta \varphi$ by Eq. (5).

$$
\Delta \varphi[\mathrm{rad}]=2 \pi \frac{\text { GPos }[\text { digital units }]}{2^{16}\left[\frac{\text { digital units }}{\text { cycle }}\right] \cdot 2^{8}\left[\frac{\text { cycles }}{\text { revolution }}\right]} .
$$

"GPos" is the grating position measurement in digital units where 0 is the "home"-position of the grating. This position is close to the homing end switch of the grating mechanism. The position counter of the Inductosyn is absolute within one cycle of $2^{16}$ digital units, corresponding to ca. $1.406^{\circ}$, where 256 (i.e. $2^{8}$ ) cycles correspond to a rotation of $360^{\circ}$. The number of cycles from the home-position is counted by an additional 5-bit counter. Therefore, the position of the grating is measured in a range from 0 to $2^{21}$, corresponding to a range of approximately $45^{\circ}$. The value of $\varphi$ at the home position is defined as $\varphi_{0}$ and is about $20^{\circ}$ by design. In the calibration model, $\varphi_{0}$ is expressed in digital units using the parameter ISOFF (Inductosyn offset). Therefore, $\varphi$ is:

$$
\varphi[\mathrm{rad}]=\Delta \varphi+\varphi_{0}=2 \pi \frac{\mathrm{GPos}+\mathrm{ISOFF}}{2^{16} \cdot 2^{8}} .
$$

The offset angle from the Littrow angle $(\gamma)$ is estimated for each channel using the optical design values of $1.2^{\circ}$ for the red channel and $0.64^{\circ}$ for the blue channel. The pixel angle $\delta$ is a function of the pixel position along a detector module. Initially, a linear model was used for this angle. In this model, the offset 8.5 is subtracted from the pixel number $(\mathrm{PN} \in[1,16])$ to make $\delta$ equal to 0 in the detector center. The result is multiplied with the pixel scale (PS). Early measurement results have shown that the calibration is more accurate if the linear model is expanded by a quadratic term as shown in Eq. (7).

$$
\begin{aligned}
\delta[\mathrm{rad}]= & (\mathrm{PN}[\mathrm{pix}]-8.5) \cdot \mathrm{PS}\left[\frac{\mathrm{rad}}{\mathrm{pix}}\right] \\
& +\operatorname{sign}(\mathrm{PN}-\mathrm{QOff}) \\
& \cdot(\mathrm{PN}-\mathrm{QOff})^{2} \cdot \mathrm{QS}\left[\frac{\mathrm{rad}}{\mathrm{pix}^{2}}\right] .
\end{aligned}
$$

In this model, the angle between two pixels increases quadratically away from a zero point. This zero point (QOff) is given as a pixel number (fractions of a pixel are allowed) at which the angle between two pixels would be equal to the pixel scale (PS). The magnitude of the quadratic increase is determined by the quadratic coefficient (QS).

The final calibration model has the parameters listed in Table 1. The first four parameters in the table are derived from laboratory measurements of specific water lines. The latter six parameters are then determined by fitting the calibration model to the results of several different water line measurements.

The groove spacing parameter $g$ and the offset angle defined by ISOFF are dependent on the position of a spaxel along the entrance slit of the spectrometer part of a specific channel. The reason for this dependence is the slit curvature (also referred to as spectral line curvature) resulting from the extended slit length (Minkowski, 1942). For the extended slit, the rays of all points that are not in the slit center (along the length of the slit) reach the grating at an angle to the plane that is normal to the grating and the grating's lines. The angle $\vartheta$ between this plane and the incoming ray changes the grating Eq. (3) resulting in Eq. (8) (Minkowski, 1942).

$$
\lambda=\frac{g_{0} \cos \vartheta}{m}(\sin \Phi+\sin \Theta) .
$$

Table 1. Wavelength calibration model parameters.

\begin{tabular}{ll}
\hline Parameter & \multicolumn{1}{c}{ Remark } \\
\hline$\lambda[\mu \mathrm{m}]$ & Position of a measured emission line as from HITRAN (2016) \\
$\mathrm{M}[1]$ & Observed order, known from instrument setting \\
GPos $[$ digital units $]$ & Result of a Gaussian fit to a line profile measured by scanning a line over a physical pixel \\
PN & Pixel number for the considered pixel \\
$g[\mathrm{~mm}]$ & Result of the calibration fit discussed below, starting value known from design \\
ISOFF $[$ digital units $]$ & Result of the calibration fit discussed below, starting value known from design \\
$\gamma[\mathrm{rad}]$ & Result of the calibration fit discussed below, starting value known from design \\
PS $[\mathrm{rad} /$ pixel $]$ & Result of the calibration fit discussed below, starting value known from design \\
QOff $[\mathrm{pixel}]$ & Result of the calibration fit discussed below, starting value 8.5 which is the detector center \\
QS $\left[\mathrm{rad} / \mathrm{pixel}^{2}\right]$ & Result of the calibration fit discussed below, starting value 10 \\
\hline
\end{tabular}


The angle $\vartheta$ can be derived from the position of a point on the slit and the focal length of the camera optics of the spectrometer. Another approach to derive $\vartheta$ is based on the slit position (SP) of the module (refer to Fig. 1) and the neutral position (NP) at which $\vartheta$ is 0 . The values of SP and NP are expressed as slit position or fractions of slit positions. The slit position, $\mathrm{SP}$ is a known value (refer to Fig. 1). The NP is a result of the calibration model fit. An additional constant "a" replaces the conversion factor from [slit position] to [mm] offset on the slit, and the focal length of the camera. The resulting equation for $\vartheta$ is:

$$
\vartheta=\tan ^{-1}\left(\frac{\mathrm{SP}-\mathrm{NP}}{a}\right) .
$$

The specific way in which the pseudo slit is constructed by the optics in FIFI-LS made it possible to counteract the slit curvature by introducing a curvature in the opposite direction within the integral field unit of the instrument (Raab, 2002). However, this measure removes the curvature for one specific wavelength only. This is understood when considering that the slit curvature introduced by the extended slit is not constant in the wavelength direction but is the result of the changing effective groove spacing $g$. By design, the wavelengths for which the curvature is eliminated within the instrument are $160 \mu \mathrm{m}$ in the red channel and $90 \mu \mathrm{m}$ in the first order of the blue channel (Raab, 2002). This approach, of counteracting the curvature within the integral field unit, had to be accounted for in the calibration model equation. This could be achieved by an extra term within the $\Phi$-angle of the incoming beam. However, the values for this term are hard to determine during a fit of the calibration model, as the parameters for the new term are not orthogonal but very close to the $\gamma$ angle and the ISOFF parameter. The model differences were less than $1 / 1000$ th of a spectral resolution element for a comparison of fits with the extra term, and fits where the additional term was absorbed into the ISOFF parameter. Therefore, the effects of the correction of the curvature are accounted for by a dependency of the ISOFF parameter on the slit position of a spaxel. Varying the ISOFF parameter for different spaxels also allows accounting for a possible tilt of the slit on the detector, and for positional variations of the individual detector modules with respect to each other resulting from manufacturing tolerances. The ISOFF differences due to manufacturing tolerances are arbitrary and therefore result in a discontinuous dependency of the ISOFF parameter on the slit position of a spaxel. This dependency cannot be described by a continuous model equation, but is the result of the fitting of the calibration model to each individual spaxel.

The calibration model parameters were fitted to the measurement data of several water lines using a two-stage process. In the first stage, a set of parameters was fitted for a calibration model of the whole detector using model Eq. (4) with 25 independent ISOFF parameters (one for each spaxel). The fitting was implemented in MATLAB ${ }^{\circledR}$ using the included "fit" function (nonlinear least squares fitting). The start values for the model parameters $g$ and $\gamma$ were derived from the instrument design. The values for ISOFF (932000ISU according to $\sim 20^{\circ}$ ), PS (6e-4 rad), QS (1e-9), and QOFF (8.5, detector center) were initial estimates.

The 25 ISOFF parameters were fitted again in the second stage individually for each spaxel, using the results of the previous stage. This improved the results because the ISOFF parameter, which also accounts for small (random) residual offsets between the detector modules in spectral direction, was fitted directly using only the data of the respective module. In this stage, a least squares fitting routine according to the approach by Stetson (1989) was implemented in MATLAB ${ }^{\circledR}$.

A typical parameter set from the latest calibration (Red Channel February 2018) is shown in Table 2 (not all 25 ISOFF parameters for all 25 Spaxels are listed). The parameters are given with as many significant digits as necessary to yield an accurate calibration. The 1-sigma accuracy intervals of the individual parameters are wider than one might expect from the number of significant digits shown in the table. But, this only reflects the fact

Table 2. Wavelength calibration model parameters for Spaxel 13 in the Red Channel from February 2018.

\begin{tabular}{|c|c|c|}
\hline Parameter & Fit result & Sigma \\
\hline $\mathrm{g} 0[\mathrm{~mm}]$ & $0.11715961 \mathrm{E}+0$ & $\pm 4.0 \mathrm{E}-7$ \\
\hline NP [slit position] & $14.3214 \mathrm{E}+0$ & $\pm 5.0 \mathrm{E}-2$ \\
\hline a [slit position] & $421.6395 \mathrm{E}+0$ & $\pm 2.7 \mathrm{E}+0$ \\
\hline ISOFF [digital units] & $1149533 \mathrm{E}+0$ & $\pm 95 \mathrm{E}+0$ \\
\hline$\gamma[\mathrm{rad}]$ & $1.6720 \mathrm{E}-2$ & fixed at design value \\
\hline PS [rad/pixel] & $5.8783 \mathrm{E}-4$ & $\pm 4.7 \mathrm{E}-7$ \\
\hline QOff [pixel] & $5.9857 \mathrm{E}+0$ & $\pm 1.5 \mathrm{E}-1$ \\
\hline $\mathrm{QS}\left[\mathrm{rad} / \mathrm{pixel}^{2}\right]$ & 1.4592 E-6 & $\pm 7.9 \mathrm{E}-8$ \\
\hline
\end{tabular}


that no single parameter can be changed in the model within its sigma corridor without affecting the optimal value for the other parameters as they are all interdependent.

After fitting the wavelength calibration model curve, two curves offset by $\pm 10 \%$ of a spectral resolution element from the fit curve were calculated for each physical pixel. The estimated instrument resolution (refer to Fig. 4) was used to calculate these offset curves. The criterion for which a calibration fit was considered sufficiently accurate was that more than $90 \%$ of the valid measurement points and fell within the allowed $\pm 10 \%$ corridor, individually for each of the 25 modules of a detector. Figure 6 shows an example for a fit curve close to the $129.339 \mu \mathrm{m}$ water line data, including the $\pm 10 \%$ corridors of allowed offsets.

The fitting process always yielded results fulfilling the accuracy criteria in the red channel. In the blue channel, the criteria were not always met. When increasing the width of the accuracy corridor from $\pm 10 \%$ to $\pm 15 \%$ of an estimated spectral resolution element, all fits in the blue channel fulfilled the accuracy criteria. The need for a wider corridor in the blue channel was caused by the true spectral resolution in the blue channel, which was considerably lower than the estimated resolution (as discussed above). The lower spectral resolution resulted in an increased uncertainty in the fits of the line positions, leading to a wider scatter in the resulting measurement points. Measured in spectral

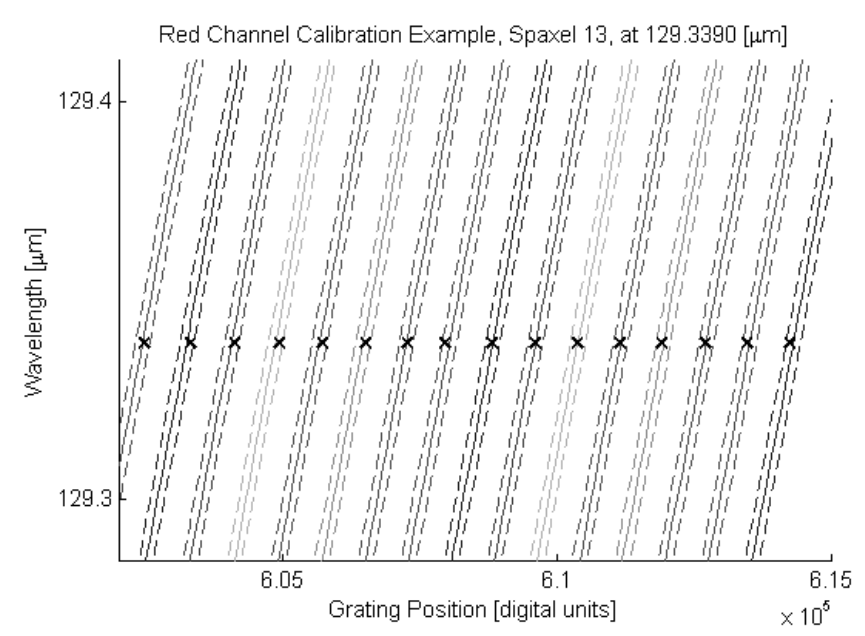

Fig. 6. Calibration curves in the red channel for the central spaxel at $129.339 \mu \mathrm{m}$. Black crosses: fitted line positions for the 16 pixels of the central spaxel. Solid lines: calibration fit results for the 16 pixels. Dashed lines: $\pm 10 \%$ of a spectral resolution element offset curves defining the goal for the accuracy corridor (Colditz, 2017). pixels, the accuracy of the spectral calibration is between about 0.15 and 0.5 pixels depending on spectral band and observed wavelength.

The calibration fits in both channels indicate that the wavelength calibration shifts by up to $50 \%$ of a spectral resolution element, for different cooldowns. These changes are most likely caused by differences in the deformations of the optical bench due to the thermal contraction during the cooldown process. Because of these changes, a new wavelength calibration, including new line position measurements is necessary for each cooldown. All parameters of the wavelength calibration model from each cooldown of the instrument are stored in the FIFI-LS data pipeline.

\section{Spatial Characterization and Calibration}

The spatial characterization of the instrument was performed using the telescope simulator described in Schweitzer (2008). A small point source was installed in the simulator that could be moved within the FOV with an xy-stage to obtain measurements for the spatial characterization of the instrument. The results of these measurements were the beam maps described in the next sub-section. Beam maps measured for different instrument settings (e.g. filters, grating position/wavelength) were used to characterize the point spread function (PSF) of the instrument as described in Sec. 3.2 and to measure the geometry of the FOV as described in Sec. 3.4. The beam map measurements also revealed the existence of spectrally shifted spatial ghosts, which are described in Sec. 3.3. In these sections, measurements will often be shown as $\mathrm{mm}$ or $\mathrm{mm}_{\text {TelSim }}$ these values are converted to seconds of arc on sky with the conversion factor 3.55 arcseconds $/ \mathrm{mm}_{\text {TelSim }}$, derived in Colditz (2017).

\subsection{Beam maps}

The main method to gather information about the spatial characteristics is the evaluation of "beam maps". A beam map represents the spatial response function of a single instrument pixel. A point source with constant intensity is used to measure a beam map, by scanning it over the whole FOV of the instrument or a part of it in discrete steps. This process yields beam maps for several or all pixels of the instrument depending on the part of the FOV that was covered by the scan. The signal received by a 


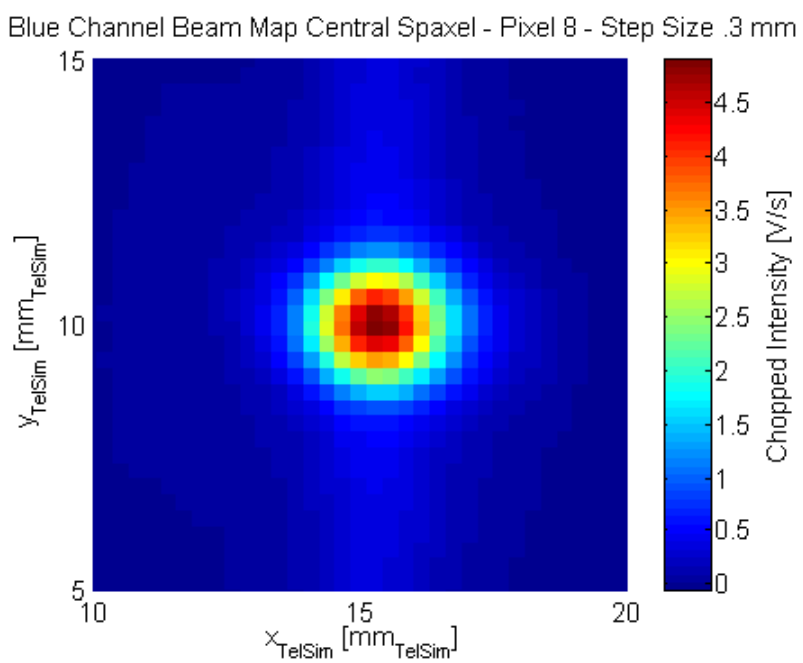

Fig. 7. Beam map for a single pixel obtained using the telescope simulator with $0.3 \mathrm{~mm}$ stepping of the point source through the FOV. The step size is measured in the telescope simulators reference frame. The result shown is for one physical (spectral) pixel within the central spaxel (Colditz, 2017).

specific pixel is plotted in relation to the point source position in the FOV using the coordinate system in which the point source was moved (e.g. the telescope simulator coordinate system). Figure 7 shows an example of a beam map for one pixel in the central spaxel of the blue channel, obtained with the telescope simulator point source and using a step size of $0.3 \mathrm{~mm}$. The point source was scanned inside out in a spiraling motion to cover the whole FOV of the pixel.

The individual "image pixels" (visible squares) in Fig. 7, should not be confused with the physical pixels of the detector or the spaxels. They represent the intensity of one measurement point for a single physical pixel at one point source position. One "image pixel" in Fig. 7, has the size of $0.3 \times 0.3 \mathrm{~mm}^{2}$, corresponding to the step size by which the point source was moved between two consecutive measurements on the spiral. The beam map of a pixel would represent its PSF projected onto the focal plane of the telescope simulator, if the point source was an ideal point source, that means infinitely small and without holding wires. In reality, the beam map represents the convolution of the pixel's PSF with the radiation from the extended point source and its holding wires. The point source is a hemisphere with a diameter of $1.5 \mathrm{~mm}$. The convolution of the PSF with the telescope simulator point source is visible in the center of Fig. 7, including the holding wires of the point source as a vertical line of slightly increased signal.
The standard step size for the point source was chosen to sample the PSF of the instrument sufficiently. Early measurements have shown that a Gaussian fit to the instrument PSF yields a FWHM of about $1.6 \mathrm{~mm}$ in the blue channel. According to the Nyquist sampling theorem, at least two measurement points per FWHM are necessary to reconstruct the Gaussian. Therefore, a standard step size of $0.6 \mathrm{~mm}$ was chosen, which includes a margin to account for changes in the size of the PSF for different wavelengths. This step size is used for the portion of the FOV that is shared by the blue and the red channel. The step size is increased to $1.2 \mathrm{~mm}$ for the portion of the FOV that is covered solely by the red channel. This increase saves time during the measurement of beam maps and is optimized for the larger FWHM of about $3.0 \mathrm{~mm}$ in the red channel.

Several beam maps have been recorded with finer step sizes of $0.3 \mathrm{~mm}$ in the portion of the FOV shared by both channels and of $0.6 \mathrm{~mm}$ in the outer portion of the FOV. These beam maps were used to identify an accurate model for the instrument's PSF. It took more than $6 \mathrm{~h}$ to complete a beam map covering the whole FOV of the instrument with the fine stepping for a single instrument setup. The fine stepping was therefore too time-consuming to be practical as the standard mode for beam map measurements.

\subsection{Instrument point spread function}

The PSF is an important property of the instrument. It is closely connected to the instrument's spatial resolution. The PSF is typically a result of diffraction processes and aberrations in the instrument optics. Its size is a function of the wavelength. For decreasing wavelengths, the size of the PSF decreases, and therefore the spatial resolution increases.

There are different ways to define the PSF for an instrument, as laid out by Anderson \& King (2000). Here, we discuss the effective PSF, which is defined as the convolution of the instrumental PSF resulting from diffraction (and optical imperfections on the optical path) with the response function of the detector pixels. The laboratory results presented here do not include the SOFIA telescope's effects on the PSF. The limiting aperture for astronomical observations is SOFIA's secondary mirror "M2". For the laboratory measurements with the telescope simulator the limiting aperture is the instrument's Lyot stop, which is approximately $13 \%$ oversized compared to the secondary mirror M2. 
Beam maps were used to identify the PSF of each pixel. Fine sampled beam maps $(0.3 \mathrm{~mm}$ and $0.6 \mathrm{~mm}$ step size) were therefore measured in both channels at one wavelength in each channel. These fine measurements were used to judge whether the PSF had unexpected features that differed from the Gaussian model used to approximate the PSF. Additionally, approximately Nyquist sampled $(0.6 \mathrm{~mm}$ and $1.2 \mathrm{~mm}$ step size) beam maps were obtained for different wavelength settings of the instrument to investigate the dependence of the spatial resolution on the observed wavelength. These beam maps were fitted using a two-dimensional Gaussian to model the telescope simulator's point source and a onedimensional Gaussian to model the thin holding wires, which connect the point source to its support frame. The combined model is shown in Eq. (10) (Schweitzer, 2008). In the equation $A_{\mathrm{ps}}$ is the peak intensity of the point source, $A_{w}$ is the peak intensity of the holding wires and $B$ is the background intensity. The free model parameters were fitted to the data using MATLAB ${ }^{\circledR}$ 's fit function employing the trust-region-reflective algorithm.

$$
\begin{aligned}
I(x, y)= & A_{p s} \cdot \exp \left[-\frac{1}{2}\left(\left(\frac{x-x_{0}}{\sigma_{x}}\right)^{2}+\left(\frac{y-y_{0}}{\sigma_{y}}\right)^{2}\right)\right] \\
& +A_{w} \cdot \exp \left[-\frac{1}{2}\left(\frac{x-x_{0}}{\sigma_{x}}\right)^{2}\right]+B .
\end{aligned}
$$

Figure 8 shows a fit of this model to a beam map of a pixel in the red channel (one physical pixel of the central spaxel) at a wavelength of $143 \mu \mathrm{m}$ with the $105 \mu \mathrm{m}$ dichroic filter. The residual plot in Fig. 9 shows how well the Gaussian model fitted the PSF.

The residual offsets between the data and the model are less than $2.5 \%$ of the peak value of the fitted Gaussian. The residuals are slightly increased toward the left corner of the plot because of a slight drop of the background. The peak on the right side of the residual plot belongs to a spatial ghost in the red channel, which is discussed further below. Generally, the residuals were less than $5 \%$ of the peak value for all pixels (excluding ghosts). The results in the blue channel were similar. Only the pixels belonging to the partially illuminated spaxels on the sides of the image slices showed residuals above $5 \%$ of the peak value. These increased residuals were mostly the result of a gradient in the background that was decreasing in the detector coordinate system's $x$-direction (i.e. parallel to the pseudo slit).

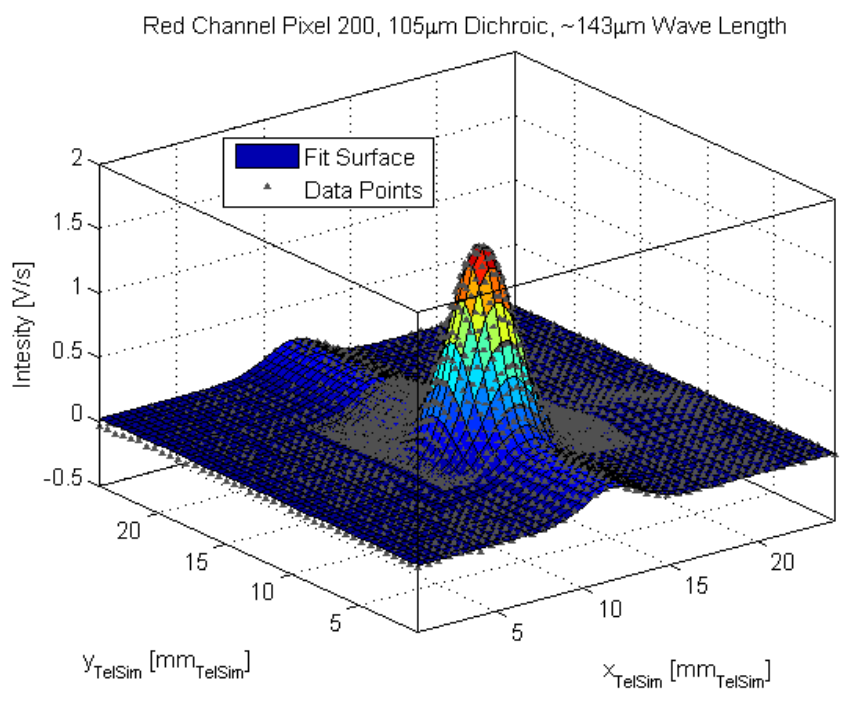

Fig. 8. 2D-Gaussian fit to the beam map data of the red channel's "central" physical pixel (a pixel in the central spaxel). The black triangles represent the measured data and the colored surface plot shows the fit result. The main peak in the center results from the pixel's PSF convolved with the telescope simulator's point source. The line of increased signal in the center along the $y$-axis is caused by the holding wires of the point source (Colditz, 2017).

The $\sigma_{x / y}$-width-parameters, resulting from the fits of model Eq. (10) to the data, were used to calculate the FWHM of the PSF, and hence the possible resolution of a sufficiently sampled observation. The FWHM is approximated by $\sigma_{x / y}$ assuming a Gaussian profile.

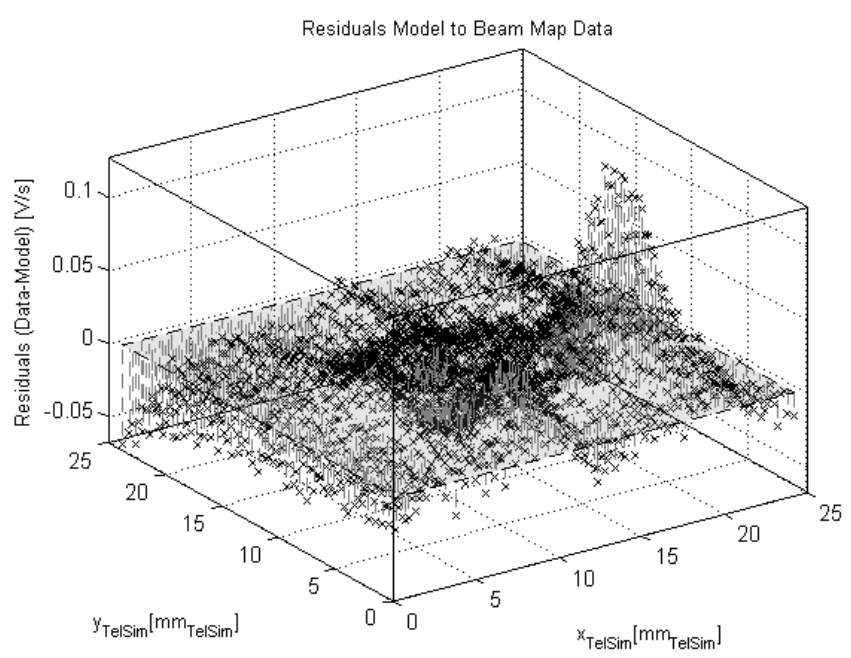

Fig. 9. Residual offsets between the fit model and data for the fit shown in Fig. 8. The low residual values in the center show that the model fits the data quite well in the area of the main peak of Fig. 8. The peak on the right side reveals that a spatial ghost is present. This means that the pixel receives light from a part of the FOV that the pixel should not be able to "see" (Colditz, 2017). 


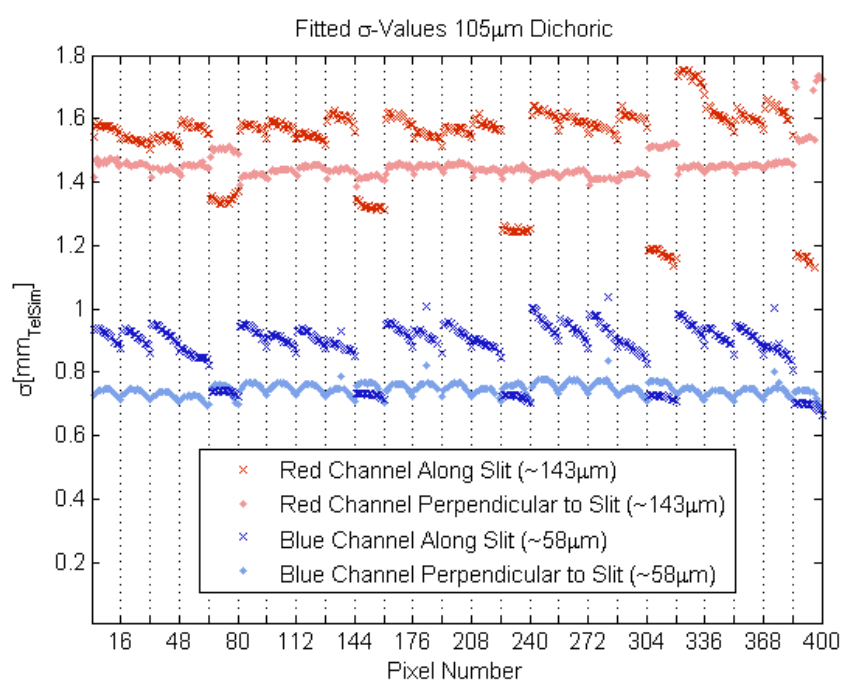

Fig. 10. $\sigma_{x, y}$-fit for all pixels in both channels for one instrument setting. The crosses indicate the $\sigma_{x}$ values and the dots the $\sigma_{y}$ values. The $\sigma_{x}$ values belonging to the pixels of spaxels $5,10,15,20$ and 25 are all smaller than those for the other pixels of a channel, indicating a residual misalignment of the pseudoslit on the detector (Colditz, 2017).

Figure 10 shows the $\sigma_{x / y}$-fit results for all 400 pixels in each channel, for one instrument setting. The $\sigma_{x}$-values describing the width of the PSF in direction along the pseudo-slit entering the spectrometer part of the instrument were higher than the $\sigma_{y}$-values describing the width of the PSF perpendicular to the slit. This observation was in agreement with the theoretical results in Raab (2002) and the results obtained in an earlier characterization measurement (Schweitzer, 2008). The figure shows that in both channels the spaxels on one side (i.e. the right side of the FOV) of each slice have $\sigma_{x}$-values that appear to be smaller than those for the other spaxels. This effect was attributed to residual misalignments of the pseudo-slits on the detectors in slit direction for both channels. These misalignments result in a partial illumination of the affected modules. The partial illumination then results in the apparent reduction of the width of the $\mathrm{PSF}$.

The standard variation of the $\sigma_{x / y}$-values over all spaxels (excluding the right column of partially illuminated spaxels) was less than 3\% for the red and less than $3.5 \%$ for the blue channel. In the red channel, the standard variation of the $\sigma_{x / y}$ values within the 16 pixels of a single spaxel was less than $2 \%$ in both directions. In the blue channel, this standard variation was about $5 \%$ for the $\sigma_{x}$-values, and the values were systematically decreasing over the detector module from pixel 1 to pixel 16. The $\sigma_{y}$-values, perpendicular to the slit, were systematically lower toward the edges of a module and had a maximum in the center. These systematic effects need further investigation and might be connected to the warped LSF presented for the blue channel in the previous section.

The mean $\sigma_{x / y}$-values over all pixels belonging to the central nine spaxels were calculated for beam maps obtained at different wavelength settings, to establish the dependency of the FWHM on the wavelength. There were two reasons to use the central nine spaxels only. The first reason was that measuring full beam maps for all 25 spaxels in both channels for multiple instrument setups was considered too time consuming. The second reason was that using the central nine spaxels avoided averaging over effects on the edge of the FOV, including the smaller apparent $\sigma_{x / y}$ values for the partially illuminated spaxels on the right side of the FOV. In some cases, the beam maps did not cover a sufficient amount of the FOV to obtain data for all nine spaxels; in these cases, the incomplete spaxels were excluded from the calculation. The FWHM values obtained with this method were corrected for the finite size (1.5 mm diameter) of the telescope simulator's point source by approximating the deconvolved FWHM.

Figures 11 and 12 show the FWHM results for the blue and the red channel in $\mathrm{mm}$ of the telescope

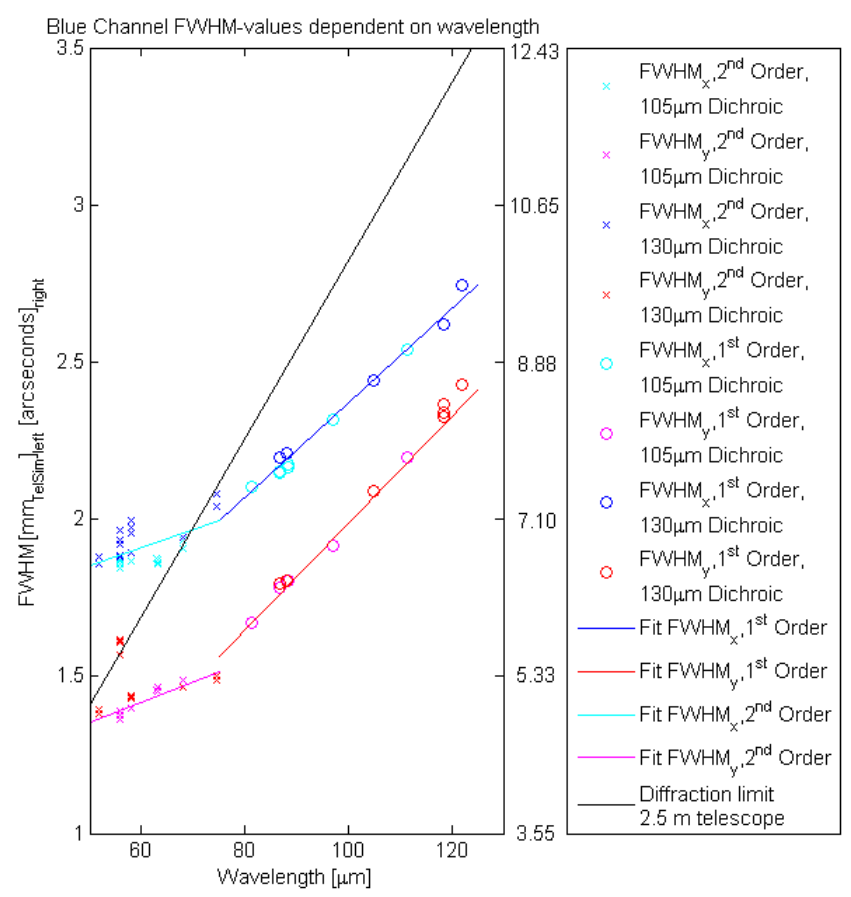

Fig. 11. The FWHM for the blue channel. The cutoff wavelength between the first and the second order is roughly $76 \mu \mathrm{m}$. The values are shown in $\mathrm{mm}$. 


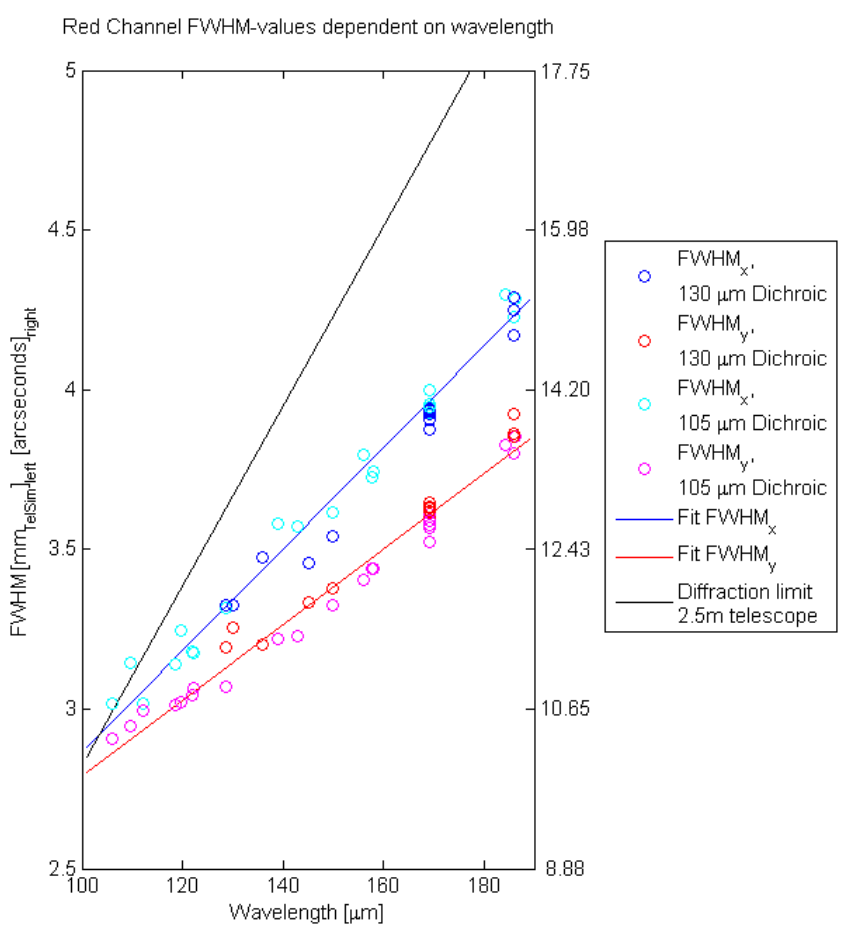

Fig. 12. The FWHM for the red channel.

simulator coordinate system and in arcseconds on sky. The derivation of the transformation coefficients from $\mathrm{mm}$ to arcseconds is presented in Colditz (2017). As discussed above, the values in the $x$-direction (along slit) were larger than the values in the $y$-direction (perpendicular to the slit).

Linear models according to Eq. (11) were fitted to the FWHM data in both directions for both channels. The parameters resulting from those fits are summarized in Table 3.

$$
\mathrm{FWHM}_{x / y}[\mathrm{~mm}]=a\left[\frac{\mathrm{mm}}{\mu \mathrm{m}}\right] * \lambda[\mu \mathrm{m}]+b[\mathrm{~mm}] .
$$

Figures 11 and 12 also show the theoretical diffraction limited FWHM of the telescope, based on the Rayleigh criterion (Hecht, 2002). This shows that the PSF of the FIFI-LS SOFIA telescope

Table 3. Parameters of the linear models for the FWHM.

\begin{tabular}{|c|c|c|c|}
\hline Channel/Order & Direction & $\mathrm{a}[\mathrm{mm} / \mu \mathrm{m}]$ & $\mathrm{b}[\mathrm{mm}]$ \\
\hline \multirow[t]{2}{*}{ Red } & Along slit & 0.0156 & 1.3214 \\
\hline & Perpendicular to slit & 0.0116 & 1.6466 \\
\hline \multirow[t]{2}{*}{ Blue $1^{\text {st }}$ Order } & Along slit & 0.0151 & 0.8621 \\
\hline & Perpendicular to slit & 0.0179 & 0.2169 \\
\hline \multirow[t]{2}{*}{ Blue $2^{\text {nd }}$ Order } & Along slit & 0.0057 & 1.5699 \\
\hline & Perpendicular to slit & 0.0064 & 1.0353 \\
\hline
\end{tabular}

combination is dominated by the telescope throughout the wavelength range of the red channel. This was expected because the instrument's Lyot stop and the instrument optics are slightly oversized with respect to the diffraction pattern expected from the telescope's limiting aperture, which is the secondary mirror. Comparing the results in the red channel for the two different dichroic filters showed that there was no significant impact of the dichroic on the FWHM $(<3 \%$ difference).

The results for the blue channel in the first order (above $76 \mu \mathrm{m}$ ) were similar to the results in the red channel for slope and dependence on dichroic. Figure 11 shows that in the second order the slope of the wavelength dependence was considerably smaller than for the first order. This difference was not expected as the optics for both orders are the same except for the different order filters, which are in a pupil position. The longest wavelength result in the blue channel's second order at $74.5 \mu \mathrm{m}$ was in agreement with the results from the first order. Towards lower wavelengths, the decrease in FWHM is not as large as expected. This effect needs further investigation. It is likely that the effect was an artifact from the finite size of the point source. The simple deconvolution procedure that was used may not be able to model the smaller FWHMs associated with the shortest wavelengths observed.

In the second order, there was also a notable difference in the values obtained for the two different dichroics. Especially for the measurement at $55.7 \mu \mathrm{m}$, the values for the $130 \mu \mathrm{m}$ dichroic were significantly increased. The reason for this effect has to be investigated. There are two possible starting points for the further investigation. The first one is that the surface of the $130 \mu \mathrm{m}$ dichroic is not perfectly flat as some distortions remained after an attempt to clean the component. The dichroic is close to a pupil position, so it might have an impact on the PSF. The second point is that the measurement at $55.7 \mu \mathrm{m}$ is within a "dip" of the dichroic's reflectivity curve. Therefore, the signal to noise ratio is about three times lower than in the measurement with the $105 \mu \mathrm{m}$ dichroic.

The results in Fig. 11 show that the FWHM of the instrument itself in the blue channel's first order was better than the theoretical diffraction limit of the SOFIA telescope. In the second order, the FWHM appeared to be higher than the theoretical diffraction limit of the telescope for the direction 
along the slit. The PSF is under-sampled in the whole spectral range of the blue channel considering the blue channel's spaxel size of $1.7 \mathrm{~mm}$. Therefore, the increased FWHM in the second order is not problematic in undithered observations.

\subsection{Spatial ghosts}

The beam map measurements discussed above also revealed spatial ghosts that are present in almost all spaxels of the red channel. A spectral analysis of these ghosts revealed that these ghosts also underwent a spectral offset. Similar effects have already been reported for the PACS instrument on Herschel, which has an integral field unit and detectors that are comparable to FIFI-LS (Vandenbussche et al., 2016). Further raytracing analysis of the FIFI-LS optics has shown that these ghosts are caused by a second pass through the spectrometer optics between the diffraction grating and preceding mirror, which is part of the collimating optics. Figure 13 shows beam maps for all 25 spaxels of the red channel.

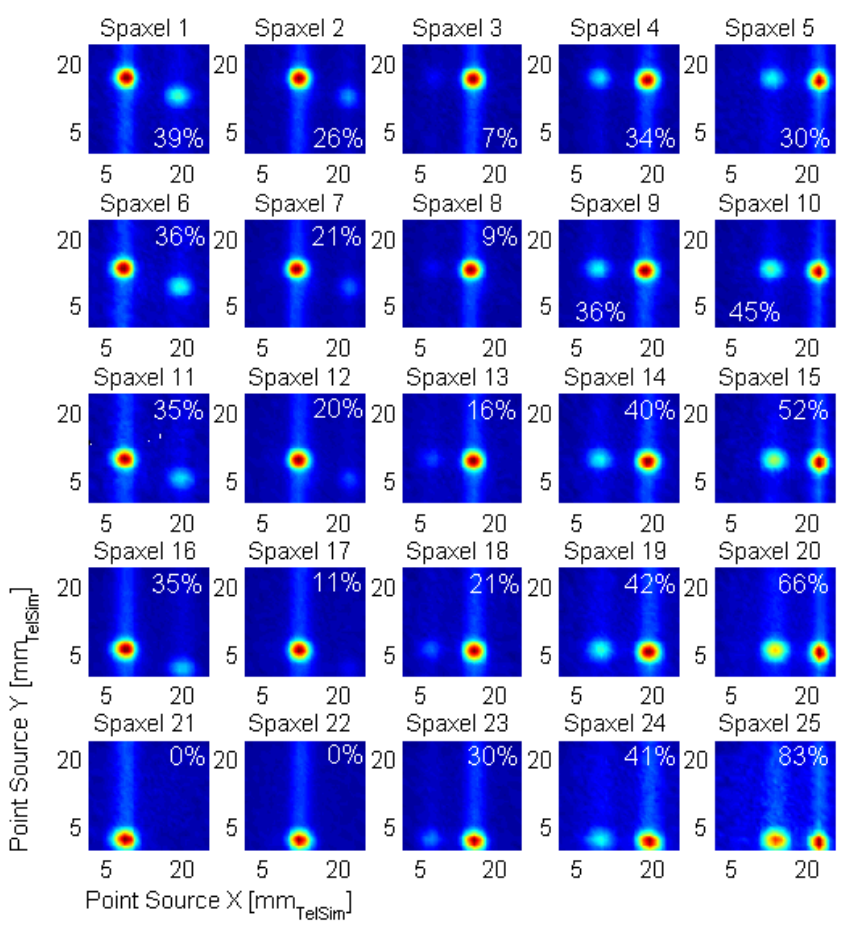

Fig. 13. Red channel beam maps for all 25 spaxels at ca. $122 \mu \mathrm{m}$. The color map is normalized to the maximum in each spaxel. The spatial ghost images of the point source are present in almost all spaxels. The \%-values indicate the intensity of a ghost peak relative to its main peak. The vertical lines crossing the peaks are caused by the signal of the holding wires of the point source in the telescope simulator (Colditz, 2017).
The main peak in each beam map in Fig. 13 corresponds to the peak position of a spaxel's response function projected onto the FOV. The lower peaks show where in the FOV light originates that causes the spatial ghosts.

The positions of the ghost peaks are the result of an offset of the slit of the ghost-light on the detector, which is schematically shown in Fig. 14.

At $122 \mu \mathrm{m}$, the ghost peaks have a relative intensity of $10-85 \%$ of the respective main peak, depending on the spaxel. An analysis determined that the light of the ghosts is spectrally offset by $15-40 \mu \mathrm{m}$ towards longer wavelengths relative to the actually observed wavelength. Therefore, the relative intensity of the ghost was decreasing for longer wavelengths on the telescope simulator's point source at room temperature (about $293 \mathrm{~K}$ ). The light of the ghosts is spread out in spectral direction, because it is not focused due to the longer optical path travelled by the ghost-light. The spectral resolution of the ghost-light is roughly a factor 10 lower than the nominal resolution of the instrument.

The ghosts do not pose a major problem for observations, as long as the observed sources do not have spectral features at the wavelength offset of the ghosts, relative to the observed spectral lines. For continuum sources it may become necessary to correct the flux for the ghosts.

\subsection{Boresight and field of view geometry}

The boresight parameters define where the instrument's individual spaxels are positioned on the sky relative to the telescope's pointing (i.e. the telescope boresight). These parameters include the spaxel positions relative to each other referenced to the central spaxel in each channel and the position of the central spaxels in reference to the telescope boresight. Knowing these parameters, their dependence on the different instrument settings and the telescope pointing, allows the calculation of the astrometry of the instrument data.

The geometry of the FOV, defined by the individual spaxel positions relative to a channel's central spaxel, was calculated based on the beam map measurement concept introduced above. A single pixel's position was measured as the position of the peak response in a beam map. The peak position was derived by fitting a Gaussian model to the beam. These fits had typical one-sigma errors of less than $5 \%$ of a spaxel size. 


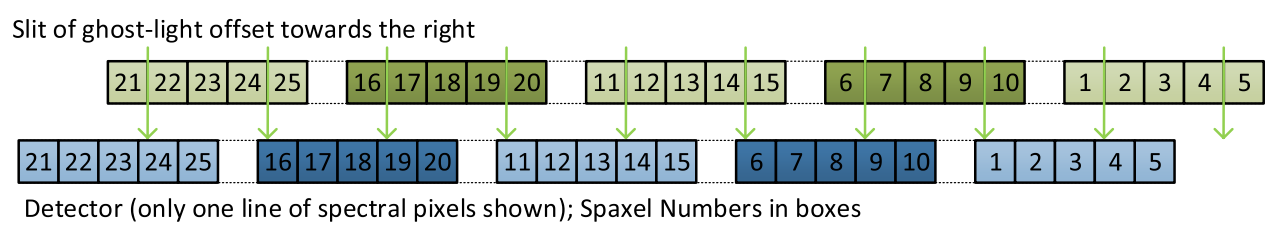

Fig. 14. Effects of the misaligned slit of ghost-light - The numbers in the boxes refer to spaxel numbers in Fig. 13. The blue boxes represent the 25 modules of the detector (one box for each spaxel). The green arrows represent incoming light from the ghost. The green boxes show how the light of the ghost is offset relative to the detector - each green box represents the ghost-light that is spatially originating from an area in the FOV that would normally belong to the spaxel number in the box; however due to the offset, the ghost-light illuminates a different spaxel number on the detector (blue boxes).

The individual spaxel positions were derived as the mean position of the 16 individual spectral pixels belonging to a specific spaxel. Generally, these 16 positions do not vary significantly $(\ll 5 \%$ of a spaxel size) in the direction perpendicular to the spectrometer slit. However, there is a systematic variation of the positions in the direction of the slit. The form of this variation points to a pincushion distortion of the image of the dispersed slit on the detector. In the most affected spaxels (on either side of the pseudo-slit) this variation has peak-to-peak offsets of $<20 \%$ of a spaxel size. These variations are not accounted for in the final spaxel position, which is a mean value of all 16 spectral pixel positions.

Figure 15 shows the relative spaxel positions for both channels. In the red channel, the grid diverts from a rectangular grid. The angle from the horizontal to the vertical in the first sector is $\sim 95^{\circ}$. The most likely reasons for this deviation are alignment offsets within the integral field unit. Because of these offsets, the positions of the five slices in the FOV vary. For example, the second slice (i.e. spaxel
6-10) is clearly offset from the general pattern by about $5 \%$ of a spaxel size to the left. These offsets are not problematic as they are known and accounted for in the data reduction process. In the blue channel, this effect is present as well, but not as strong. In the blue channel, the spaxels on the right side $(5,10,15,20$ and 25) appear too far left compared to a regular grid. The reason for this is a residual misalignment of the whole slit on the detector resulting in a partial illumination of the affected spaxels. This effect is also present in the red channel but not as severe. The numerical values for the relative spaxel positions are stored in the FIFI-LS data pipeline and are used during the data reduction process to calculate the astrometry of the individual spaxels.

Using the relative spaxel positions, the average spaxel distances were calculated. The results are summarized in Table 4 . These values are rough estimates of the spaxel size. The actual spaxel size is smaller because at the edges of the spaxels light is not detected due to the spacing between individual physical pixels. The mean distance between spaxels
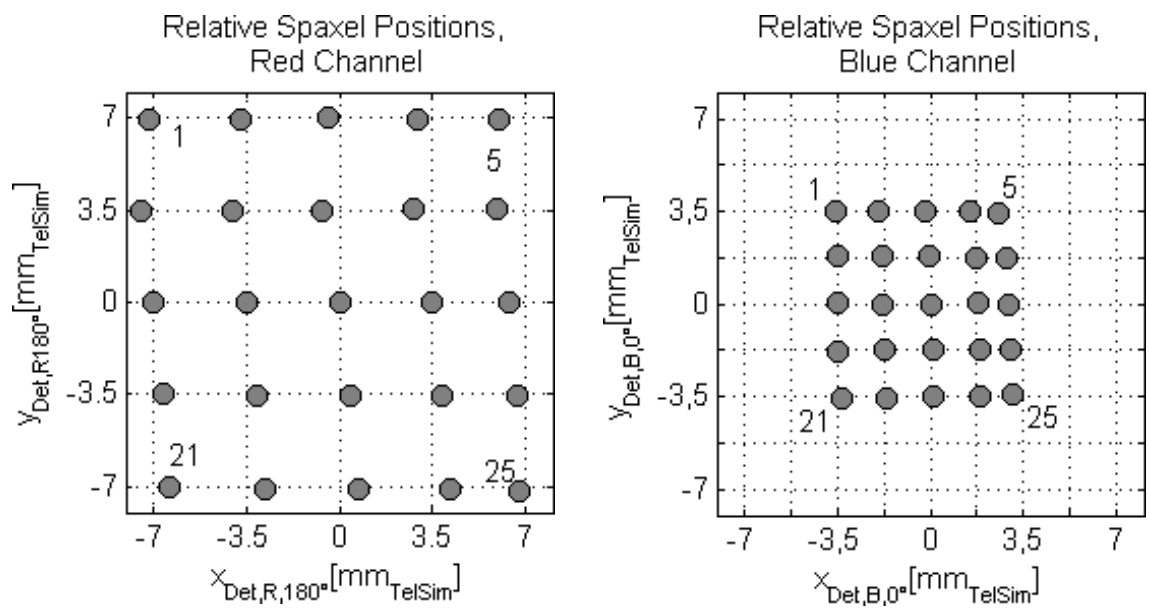

Fig. 15. Relative spaxel positions for both channels in their respective coordinate systems. The corner spaxels are numbered for orientation (Colditz, 2017). 
Table 4. Spaxel distances.

\begin{tabular}{lcccc}
\hline & \multicolumn{2}{c}{ Red channel } & \multicolumn{2}{c}{ Blue channel } \\
\hline $\begin{array}{c}\text { Horizontal } \\
\text { Distance }\end{array}$ & $3.44 \mathrm{~mm}$ & $12.2 \operatorname{arcsec}$ & $1.73 \mathrm{~mm}$ & $6.14 \operatorname{arcsec}$ \\
$\begin{array}{c}\text { Horizontal } \\
\text { Distance } \sigma\end{array}$ & $0.079 \mathrm{~mm}$ & $0.28 \operatorname{arcsec}$ & $0.048 \mathrm{~mm}$ & $0.17 \operatorname{arcsec}$ \\
$\begin{array}{c}\text { Vertical } \\
\text { Distance }\end{array}$ & $3.52 \mathrm{~mm}$ & $12.5 \operatorname{arcsec}$ & $1.76 \mathrm{~mm}$ & $6.25 \operatorname{arcsec}$ \\
$\begin{array}{c}\text { Vertical } \\
\text { Distance } \sigma\end{array}$ & $0.049 \mathrm{~mm}$ & $0.17 \operatorname{arcsec}$ & $0.045 \mathrm{~mm}$ & $0.16 \operatorname{arcsec}$ \\
\hline
\end{tabular}

of one slice in slit-direction $\left(x_{\mathrm{det}}\right)$ was calculated to derive the horizontal spaxel distances shown in Table 4. The spaxels on the far right of a slice were not used for this calculation. The vertical distance between the spaxels, was calculated using the mean values of the position perpendicular to the slit $\left(y_{\text {det }}\right)$ over each slice and then using the mean vertical distances between the slices.

The effects of different instrument settings (e.g. filters, grating position/wavelength) on the relative spaxel positions have also been investigated. In the red channel, the positions are not affected significantly by different settings. The changes of the positions are less than $5 \%$ of a spaxel size, which is comparable to the one-sigma error of the position fits. In the blue channel, the relative positions of the spaxels of the outer columns (left and right side) are shifted less than $10 \%$ of the spaxel size by the filter setting and by less than $20 \%$ of the spaxel size by the grating position/wavelength. These shifts are attributed to slight variations of the slit position on the detector, which affect the partial illumination especially on the spaxels in the right column.

The models and procedures used to describe the offset of the central spaxels in the blue and red channel from the telescope boresight have been presented in Colditz et al. (2014). This includes the effects of the rotation of the instrument's K-Mirror, which is used as a beam rotator to select and hold an optimal orientation of the sky in the instrument's FOV. The parameters of these models are also stored in the FIFI-LS data pipeline.

\section{Summary}

The spectral and spatial characteristics of FIFI-LS have been investigated using laboratory measurements employing a specialized telescope simulator for the instrument. The investigation of the spectral characteristics has shown that the instrument can be calibrated to better than $10 \%$ of a spectral resolution element over all spectral bands. The effective spectral resolution of the instrument is lower than initial estimates. In the red channel, this may be caused by a slight defocus of the dispersed light in spectral direction on the detector, while still having a Gaussian LSF. In the blue channel, the lower resolution is caused by deviations from the expected Gaussian LSF, the cause of which is yet to be fully investigated.

The investigation of the spatial characteristics shows that the instrument's intrinsic PSF is smaller than the PSF from the SOFIA telescope for most of the spectral range. Therefore, the telescope PSF should be the dominating factor for the effective spatial resolution of FIFI-LS on SOFIA. The PSF of the instrument is well modeled by a Gaussian, without strong deviations. Most of the spaxels in the red channel receive light from a spectrally shifted spatial ghost. Since the root-cause of this ghost has been found, it will be possible to model the ghost-light and minimize its impact on reduced data if necessary. This correction is not yet included in the FIFI-LS data pipeline. The geometry of the focal plane of FIFI-LS has been measured in the laboratory and on sky. The results are used to calculate the astrometry of the reduced data correctly.

\section{Acknowledgments}

SOFIA, the "Stratospheric Observatory for Infrared Astronomy" is a joint project of the Deutsches Zentrum für Luft- und Raumfahrt e.V. (DLR; German Aerospace Centre, grant: 50OK0901) and the National Aeronautics and Space Administration (NASA). It is funded on behalf of DLR by the Federal Ministry of Economics and Technology based on legislation by the German Parliament, the state of Baden-Württemberg, and the Universität Stuttgart. Scientific operation for Germany is coordinated by the German SOFIA-Institute (DSI) of the Universität Stuttgart, in the USA by the Universities Space Research Association (USRA). The commissioning of FIFI-LS was supported by the Bundesministerium für Wirtschaft und Technologie (Federal Ministry of Economics and Technology of the Federal Republic of Germany) through the DLR Space Administration (grant: 50OK1201). The team would like to thank the SOFIA observatory including NASA, USRA, DLR and DSI. FIFI-LS's commissioning as facility science instrument was a 
complete success only due to the excellent cooperation of all participants.

\section{References}

Anderson, J. \& King, I. R. [2000] "Toward high-precision astrometry with WFPC2. i. Deriving an accurate point-spread function," PASP 112, 776.

Colditz, S., Klein, R., Beckmann, S., Bryant, A., Fischer, C. et al. [2014] "Boresight calibration of FIFI-LS: In theory, in the lab and on sky," in Proc. SPIE 9147, Ground-based and Airborne Instrumentation for Astronomy $V$, 91474S.

Colditz, S. [2017] FIFI-LS - A Field-Imaging Far-Infrared Line Spectrometer for SOFIA: Completion of the Instrument, Laboratory and In-flight Calibration and Characterization (Dr. Hut Verlag), Dissertation, University of Stuttgart.

Erickson, E. F. \& Davidson, J. A. [1993] "SOFIA: Stratospheric observatory for infrared astronomy," Adv. Space Res. 13(12), (12)549-(12)556.

Fischer, C., Beckmann, S., Bryant, A. et al. [2018] "FIFI-LS: The field-imaging far-infrared line spectrometer on SOFIA," J. Astronom. Instrum. 7, 1840003.

Hecht, E. [2002] Optics (Addison Wesley).

HITRAN [2016] HITRAN on the Web, http://hitran.iao.ru, Harvard-Smithsonian Center for Astrophysics (CFA), Cambridge, MA, USA, V.E. Zuev Institute of Atmospheric Optics (IAO), Tomsk, Russia [accessed 24 March 2016].
Krabbe, A., Mehlert, D., Röser, H.-P. \& Scorza, C. [2013] "SOFIA, an airborne observatory for infrared astronomy," Eur. J. Phys. 34/6/, S161-S177.

Minkowski, R. [1942] "Curvature of the lines in plane-grating spectra," Astrophys. J. 96, 306.

Raab, W. [2002] Entwicklung und Bau des Ferninfrarot-Spektrometers FIFI LS und ISO-Beobachtungen des galaktischen Zentrums, Dissertation of the Faculty of Physics, LudwigMaximilian-University, Munich.

Schweitzer, M. [2008] Optical Alignment and Characterization of FIFI-LS - the Field-Imaging Far-Infrared Line Spectrometer, Dissertation, Faculty of Physics, Ludwig-Maximilian-University, Munich.

Stetson, P. B. [1989] The Techniques of Least Squares and Stellar Photometry with CCDs, Lecture Series, http:// ned.ipac.caltech.edu/level5/Stetson/Stetson_contents.html [accessed 28 May 2015].

Voigt, H. H. [2012] "Abriss der Astronomie," In Wesentlich überarb. u. erw. Auflage, eds. Röser, H. J., Tscharnuter, W. (Wiley-VCH), pp. 269-271.

Vandenbussche, B., Contursi, A., Feuchtgruber, H. et al. [2016] PACS Spectroscopy performance and calibration, PICC-KLTN-41 http://herschel.esac.esa.int/twiki/bin/view/Public/ PacsCalibrationWeb [accessed 17 July 2018].

Young, E. T., Becklin, E. E., Marcum, P. M. et al. [2012] "Early science with SOFIA, the stratospheric observatory for infrared astronomy," Astrophys. J. 749, L17. 